\title{
Influence of sensory manipulation on postural control in Parkinson's disease
}

\author{
J A Waterston, M B Hawken, S Tanyeri, P Jäntti, C Kennard
}

\begin{abstract}
Postural control was assessed on a tilting platform system in 20 patients with idiopathic Parkinson's disease and 20 agematched controls. The amount of information provided by vision and lower limb proprioception was varied during the experiment to investigate the influence of changes in sensory cues on postural control. The patient group with clinical evidence of impaired postural control (Hoehn and Yahr III) had significantly higher sway scores over all sensory conditions than either the Hoehn and Yahr II group or controls. The pattern of sway scores indicated that no obvious deficit in the quality, or processing, of sensory information was responsible for the postural instability observed in this group. The patients in both Hoehn and Yahr groups were also able to respond appropriately to potentially destabilising sensory conflict situations and significantly improved their sway scores when provided with visual feedback of body sway. The results indicate that in Parkinson's disease, the main site of dysfunction in postural control is likely to be at a central motor level.
\end{abstract}

$(\Im$ Neurol Neurosurg Psychiatry 1993;56:1276-1281)

Postural instability is a major clinical problem affecting patients with Parkinson's disease and usually becomes manifest in the later stages of the disease when it is often relatively resistant to drug treatment. Several authors have described abnormalities of postural reflexes ${ }^{1-11}$ which may account for some or all of the observed clinical postural deficits. The process of maintaining balance also involves the use of visual, proprioceptive, and vestibular information, and sensory information must be integrated with the internally specified set of goals related to the motor task'12 before appropriate motor commands can be specified. This process can involve arbitration in cases of sensory conflict, and short or long term adaptive responses to loss of sensory function and to changes in the requirements of the task.

The degree to which sensory integration is impaired in Parkinson's disease has rarely been studied. Bronstein et al ${ }^{13}$ reported that some patients had difficulty in suppressing the destabilising postural activity induced by repeated exposure to a moving visual scene, in contrast to normal controls and patients with cerebellar disease who adapted quickly after the first stimulus. Two studies have also suggested that patients with parkinson's disease may be less accurate than control subjects in assessing the direction of the visual ${ }^{14}$ and postural ${ }^{15}$ vertical.

A common clinical observation is the improvement in motor performance produced by manipulation of sensory input, as in the use of auditory stimuli or lines drawn on the floor to overcome freezing of gait. ${ }^{1}$ This phenomenon suggests that parkinsonian subjects are able to use sensory cues to overcome difficulties in initiation or continuation of movement. Normal subjects can use the information provided by visual feedback of sway movements to reduce body sway. ${ }^{16}$ The effect of sensory cues, in particular visual feedback, has not been examined in the context of postural motor control in Parkinson's disease, although inability to use such information to reduce body sway has been found in older subjects, some of whom also showed clinically impaired control of posture. ${ }^{16}$

We assessed postural sway in control subjects and parkinsonian patients to document any postural instability, and to study its relation to disease severity. In addition, we investigated the relative importance of vision and lower limb proprioception in the control of sway by varying the amount of sensory information available to the subject, based on the method developed by Nashner. ${ }^{17}$ Some of the manipulations of sensory information exposed the subjects to situations involving sensory conflict, providing us with the opportunity to evaluate the central integrative functions involved in postural control. The effect of sway feedback was also examined to see whether this information could be used by parkinsonian subjects to improve sway performance.

\section{Subjects and methods}

SUBJECTS

Twenty men with idiopathic Parkinson's disease and 20 age-matched male controls were studied. The diagnosis of idiopathic disease was made on the basis of clinical findings and response to levodopa. All subjects were assessed clinically and were excluded if evidence was found of other significant pathology that might affect postural control, such as vestibular disease, reduced visual acuity, or neuromuscular dysfunction. An assessment of disease severity, using the Hoehn and $\mathrm{Yahr}^{18}$ 
rating scale, was carried out by one of the investigators just before testing. There were nine patients with Grade II (HY2) and 11 with Grade III disease (HY3). None of the subjects exhibited significant body tremor that might have affected measures of sway. The mean age of the patients was 63.8 years (range 41-78), and that of the controls 62.7 years (range 45-74). Patients were instructed to withhold their anti-parkinsonian medication on the day of testing if possible, but not if this produced disabling motor symptoms. A total of 18 patients were taking medication, the remaining two being recently diagnosed and drug free. Informed consent was obtained, and the experiments were approved by the local ethics committee.

\section{APPARATUS}

A schematic diagram of the apparatus is shown in fig 1. Subjects stood on a platform that could be rotated about the axis of the ankle joints by an electrohydraulic servo. Curtains with a red and white chequered pattern were positioned one metre on each side of the subject to provide a structured visual environment, and an oscilloscope screen was positioned one metre in front of the subject at eye level in the centre of a black curtain. Handrails were mounted on either side of the platform to provide support for the subject in the event of loss of balance. Sway angle was measured by a light rod attached at waist level, which was connected to a linear transducer (LVDT, RDP Electronics type D5/500E, linearity $0 \cdot 1 \%$ FSD). The axis of rotation of the vertical lever was set at ankle height, so that its angular displacement matched that of the subject. The signal from the linear transducer represented sway angle with an error of less than $0.5 \%$ over $10^{\circ}$ up or down.

\section{PROCEDURE}

Subjects stood without shoes on the platform

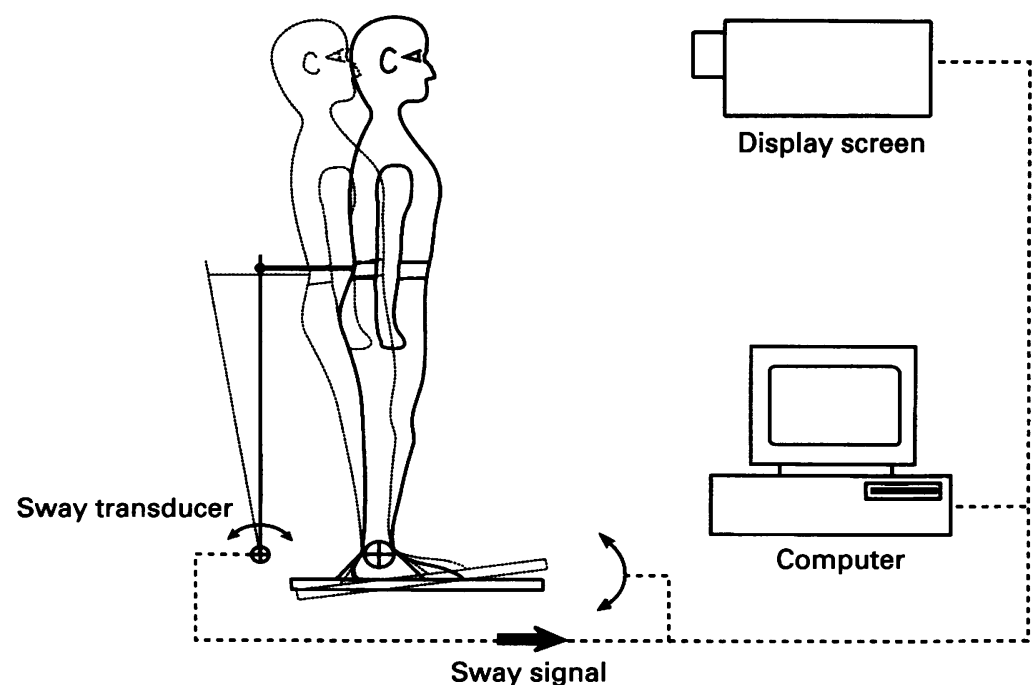

Figure 1 Schematic diagram of the apparatus. A sway transducer measured anteroposterior sway which was analysed off line by computer. The sway signal was used to drive the supporting platform during the ankle stabilised conditions, or a moving horizontal line on the display screen during the sway feedback conditions. See text for further details. with feet a comfortable distance apart. They were asked to control body sway primarily at the ankle joints, keeping the hip and knee joints as straight as possible. A period of practice was allowed, usually about $30 \mathrm{~s}$, until the subject became familiar with each test condition: and short rest periods were given between each test.

Six possible combinations of test conditions were available, using three visual conditions: eyes open (EO); eyes closed (EC); and sway feedback (SF), and two proprioceptive conditions: fixed platform (FP) and ankles stabilised (AS).

During sway feedback the sway signal from the transducer was displayed on the oscilloscope as a horizontal line which moved up with forward sway and down with backward sway. The subject was asked to keep the moving line level with a horizontal reference point, which was set at a comfortable level before the test. Gain was set to produce one degree of displacement of the line on the retina for one degree of sway as previous tests had shown this to be effective in reducing sway in control subjects. The three visual conditions were presented both with the platform fixed, and with the sway signal controlling platform movement (ankles stabilised). The gain was set so that the platform rotation followed the subject's movements as closely as possible. This minimises ankle joint rotation, and reduces proprioceptive input from the ankle joints and muscles to a very low level ${ }^{17}$ without causing a major increase in platform compliance, which would otherwise reduce the effectiveness of compensatory postural adjustments. The order of presentation of the six test conditions was varied between subjects to compensate for fatigue, practice and carry over effects.

For each trial, the sway signal was low pass filtered to prevent aliasing ( 3 pole Butterworth analogue LPF, $-3 \mathrm{~dB}$ at $10 \mathrm{~Hz}$ ), and digitised for $80 \mathrm{~s}$ at $20 \mathrm{~Hz}$ (Digitimer D210 12-bit A to D card). Subsequently, $60 \mathrm{~s}$ of the sway signal was selected manually on the computer screen for analysis and the root mean square (RMS) amplitude was calculated. In cases where subjects lost balance no sway score was available, and in order to include these subjects in the analysis it was necessary to find some method of estimating an appropriate score (omitting such subjects would bias the analysis). We used a method similar to that described by Nashner et al. ${ }^{17}$ Subjects who lost balance were allocated a sway amplitude equal to that of the subject (patient or control) who had the largest RMS sway amplitude in any condition without losing balance. This procedure necessarily underestimates the sway in those tests where subjects lost balance.

Independent of this, the distribution of RMS sway amplitude values was found to be skewed, with the median shifted towards the lower scores. A natural log transform was therefore applied before analysis to normalise the data so that a factorial repeated measures analysis of variance could be performed. 
Repeated measures (within subject) factors were vision (EC, EO, SF) and platform condition (FP, AS). The between subjects factor was subject group (control, HY2, HY3). The number of times subjects in the different groups lost balance with AS + EC were compared using Fisher's exact test.

\section{Results}

The results for each of the three subject groups obtained under the six test conditions are plotted in fig 2 . The analysis of variance showed a significant effect of subject group $(p=0.0045)$. Unplanned comparison of the means over all test conditions for the three subject groups using the Student-NewmanKeuls method showed that the HY3 patients had larger sway scores overall $(p<0.05)$ than the HY2 patients and the controls, who did not differ (fig 3 ).

With the platform fixed, the mean sway scores of the HY2 group were identical to those of the controls under all visual conditions, whereas the mean scores for the HY3 group were larger. Under the AS condition the three groups were separated, the HY3 group having larger mean scores than the HY2 group and controls. In both patient subgroups there was a large variance in the sway scores which reflected wide differences in performance, with some subjects in both the Hoehn and Yahr groups exhibiting normal sway values.

This was not the case for the AS + EC condition, where most HY3 subjects lost balance either before or during the measurement. The table shows the numbers of subjects who lost balance in the three subject groups. Fisher's exact test of the differences

Figure 2 Means of transformed sway scores versus test conditions for the controls (O) and Parkinson subjects (( $\mathbf{\square})$ Hoehn and Yahr group II; (A) group HI) (error bars $\pm 1 S D$. $. E C=$ eyes closed; $E O=$ eyes open $S F=$ sway feedback.

Figure 3 Main effect of subject group ( $p=$ $0.0045)$. Mean sway for Hoehn and Yahr group III (HY3) greater than that for controls and Hoehn and Yahr group II (HY2) $(p<0.05)$. (Error bars $\pm 1 S D)$.
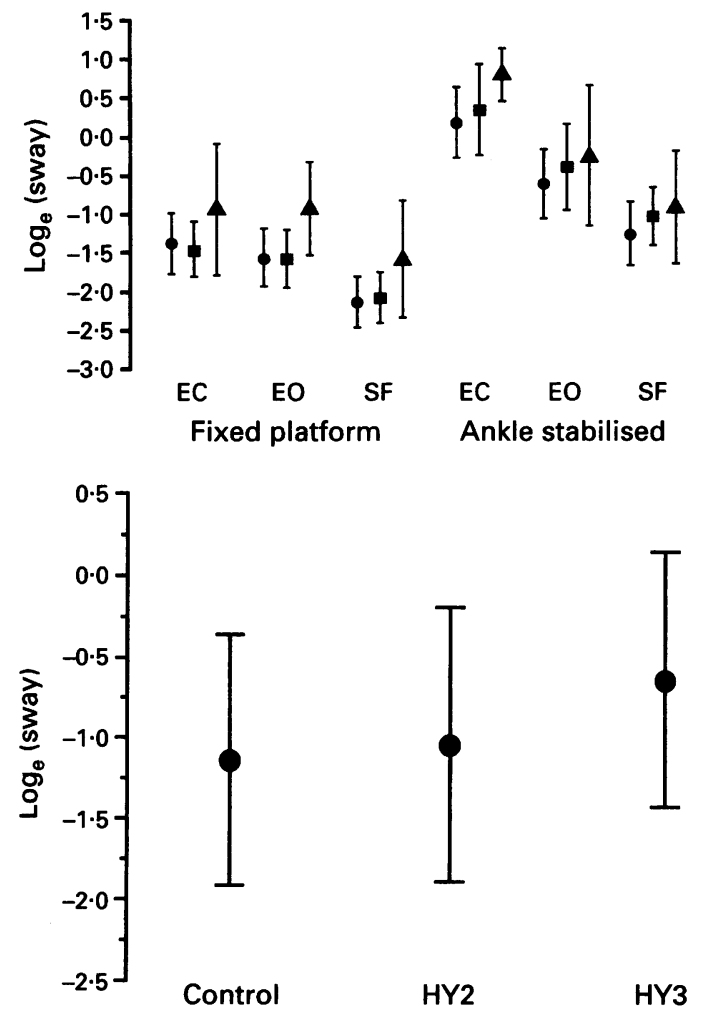

in the incidence of loss of balance gave a twotail $p$ value of 0.00097 for this $3 \times 2$ table. To examine the differences between the groups, the table was partitioned into two independent $2 \times 2$ tables using the method described in Siegel and Castellan. ${ }^{19}$ The first table compared controls with HY2 subjects, and the rates of balance loss did not differ significantly $(p>0 \cdot 1)$. The second table combined controls and HY2 subjects and compared the combination with HY3. The difference in rates of loss of balance was statistically significant $(p<0.001)$. The $3 \times 2$ table was partitioned in this way because HY3 subjects have a known, prior, clinically demonstrable deficit in postural control and, in this respect, differ from both HY2 subjects and controls. Losses of balance in the HY3 group correlated with the reported incidence of falls; seven out of eight patients who had reported falls lost balance during the tests.

There was a significant interaction between the visual and proprioceptive conditions, independent of group, which indicates that subject's responses to changes in visual condition when the platform was fixed differed from those under ankle stabilised conditions (fig 4). A contrast analysis applied to this interaction showed a significantly larger increase in sway score in response to eye closure with ankle stabilisation $(p<0.0001)$, whereas the reduction in sway score with sway feedback did not differ significantly between the two platform conditions ( $p=$ 0.4858 ). The figure also shows that sway scores were higher in all cases with ankle stabilisation, a result which was again significant (platform, main effect, $\mathrm{p}<0.0001$ ).

To investigate whether disturbance of vestibular function was a possible cause of impaired balance in our patient group, caloric testing was performed on six of the 13

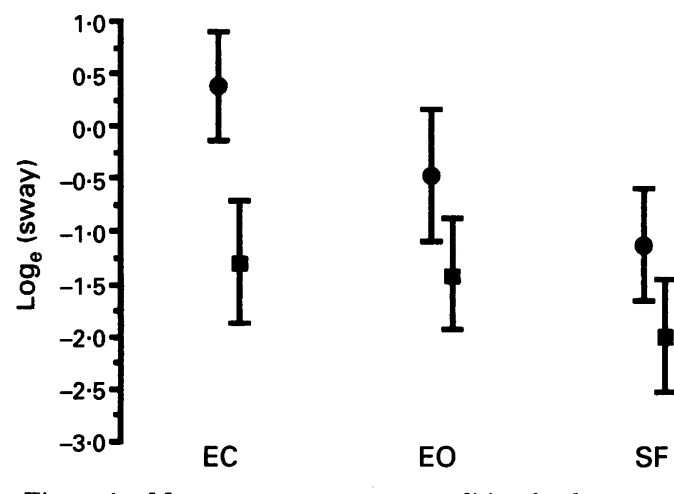

Figure 4 Mean sway scores $v$ test condition for data collapsed over all three subject groups to show the vision $\times$ platform interaction $(p<0.0001$ ). (O) ankles stabilised; (D) fixed platform, $E C=$ eyes closed. $E O=$ eyes open 
Parkinsonian patients and two of three controls who lost balance during the AS + EC condition. Abnormalities were found in two patients. Both had evidence of a partial, unilateral canal paresis (defined as a greater than $20 \%$ asymmetry on maximal slow phase velocity criteria), without significant directional preponderance. Neither had evidence of bilateral hypofunction.

\section{Discussion}

In our patient group we were unable to demonstrate any major abnormalities attributable to dysfunction in the sensory pathways that are important for postural control. The patients' ability to respond in an appropriate manner to the sensory conflict situations in these experiments also indicated that the central integrative functions were intact. Rather, the increase in sway seen under all platform conditions is consistent with the conclusion that the main pathology is confined to the pathways responsible for postural motor control.

Control of balance while standing is a complex process involving the integration of visual, vestibular, and proprioceptive information. The relative priority given to information from the different sensory systems can be varied by the use of internal weighting. Misleading sensory input can usually, but not always, be suppressed in normal subjects if sensory conflicts occur, both during transient $^{20-22}$ and in steady state conditions. ${ }^{23}$ The contribution of visual information to control of balance depends on the details of the visual environment and on the requirements of the balancing task. Vision seems to contribute minimally to the maintenance of balance during normal standing, but assumes an increasingly important role when the balancing task becomes more difficult, or when input from the other two sensory systems is reduced. ${ }^{24-26}$ This can be demonstrated clinically by a positive Romberg test in a patient with reduced proprioceptive function, and also by the significant vision/proprioception interaction in our study, where loss of visual information had a larger effect on sway scores in the absence of proprioception. This redundancy of sensory input under normal conditions appears to allow the best possible control under conditions where sensory cues are reduced. In cases of sensory conflict, however, the position is different: the postural control system must decide which sensory input or inputs are providing reliable information. There is some evidence to suggest that vision may be preferred, and even that incorrect visual cues may be used despite the presence of accurate information from the other senses. ${ }^{20}$

A number of studies have suggested that most, if not all, of the postural abnormalities in Parkinson's disease are due to ineffective selection or programming of postural reflexes. Abnormalities in the amplitude, duration, and coordination of the long latency reflexes, 258927 anticipatory postural responses, ${ }^{3610}$ and postural strategies, ${ }^{411}$ have all been described. In contrast to this, a number of potentially relevant sensory abnormalities have been documented. Abnormalities of sensory perception are sometimes apparent, as in the judgement of the visual vertical when the body is upright or tilted, ${ }^{14} 15$ and of the postural vertical after a prolonged body tilt. ${ }^{15}$ Clinical observations demonstrate that sensory stimuli can also have a powerful effect on movement in Parkinson's disease. ${ }^{1}$ Patients may freeze suddenly while walking through a doorway (akinesia paradoxica), and conversely, visual or auditory stimuli may be used to unlock the frozen patient.

The HY3 group is distinguished clinically by the finding of postural instability, and it was therefore not surprising that the HY3, but not the HY2 group, was significantly different from controls on the basis of the sway scores. Figure 2 suggests that there may be a differential effect of ankle stabilisation in the HY2 group but there is no statistical confirmation of this (platform $\times$ group interaction, $p=0 \cdot 18$ ). It is reassuring that the clinical assessment of postural control can predict platform performance of the group as a whole, but at present the degree of intersubject variability precludes the use of this investigative technique as a predictive test of postural instability in individual patients, at least on the basis of one set of sway recordings.

The finding that many patients were unable to complete the AS + EC test suggested that impairment of vestibular function may have been responsible for the postural instability in this group as performance on this subtest relies heavily on vestibular information. ${ }^{17}$ Some authors have demonstrated evidence of reduced vestibular function in parkinsonian patients, ${ }^{28}{ }^{29}$ whereas others have found no abnormalities. ${ }^{2730}$ Most of the vestibular function tests performed on our group were within normal limits, only two of the patients tested having a significant unilateral reduction in vestibular function. In view of the fact that they did not complain of any symptoms of vestibular origin (other than postural instability), it can be assumed that the abnormalities found on testing were probably long standing and therefore well compensated. It has been shown that compensation following unilateral vestibular lesions is associated with resolution of posturographic abnormalities in most patients, ${ }^{31-33}$ so the abnormalities in our patients are of doubtful clinical significance in the explanation of postural instability. In support of this it has been demonstrated that abnormalities in postural reactions in standing patients with Parkinson's disease were unlike those in subjects with overt loss of vestibular function. ${ }^{1127}$ An alternative explanation for the large number of patients who lost balance during the AS + EC condition is that their increased sway amplitude produced by the most difficult test condition brought them nearer to or beyond their limits of stability. Another possibility is that patients were unable to adapt 
quickly to the demands of an unfamiliar and challenging task. ${ }^{23} \mathrm{We}$ did not investigate this possibility as it was not the aim of the study to examine adaptive or learning effects, but such effects will need to be explored before reliable use of these techniques for diagnosis or research is possible.

Our patients showed neither clinical evidence of proprioceptive deficits, nor disturbance of visual acuity, although specific tests of visual motion detection and contrast sensitivity were not performed. Reduced visual contrast sensitivity has been reported in Parkinson's disease, ${ }^{34-36}$ but this is unlikely to affect control of posture. Visual control of posture and gait appears rather to depend on the information provided by the continuous distortion and flow (produced by motion) of the image of the environment over large areas of the retina,${ }^{37}$ and no studies of the response of Parkinsonian subjects to this type of stimulus have been reported.

Several studies have suggested that patients' performance in certain motor tasks, such as manual tracking ${ }^{38-40}$ and saccadic eye movement generation, ${ }^{41}$ is worse than that of controls when direct visual cues are absent, and Bronstein $e t a l^{13}$ have reported that patients were unable to adapt in order to suppress the destabilising postural activity produced by a misleading visual stimulus when the stimulus was presented several times. These findings imply that patients may be especially sensitive to loss of visual cues, and that they may be unable to disregard inappropriate visual information in cases of sensory conflict, possibly because the internal weighting mechanisms which suppress unwanted or inappropriate sensory input during conflict situations may have been disturbed.

We found no evidence that parkinsonian patients are either more reliant on vision, or are any less able to suppress sensory conflicts than controls. In our experiments the ankle stabilised conditions introduce a potential conflict between proprioceptive information, which indicates minimal sway, and visual and vestibular cues which register increased sway. This mismatch is successfully managed by both controls and patients, all of whom make corrections using visual and vestibular sensory information, although the lack of proprioceptive information worsens sway control in both groups. This result suggests that the internal weighting mechanisms are intact in Parkinson's disease, but we did not assess the response to misleading visual information in this study. In view of the report from Bronstein et al, ${ }^{13}$ it might be concluded that the abnormalities induced as a result of sensory conflict are only apparent when inappropriate visual cues are used, perhaps reflecting the apparent tendency to accept visual cues as correct when they disagree with information from the other senses. ${ }^{172}$ Under our test conditions, however, performance was still impaired and falls still occurred in the HY3 group, even in the absence of vision, suggesting that misleading visual input is not absolutely necessary for the generation of postural instability in parkinsonism.

Our results have shown that patients, chiefly the HY3 group, are more unsteady than controls over all conditions tested, whatever the nature of the sensory cues, suggesting that the main disability affecting postural control in these patients is at the level of central motor processing. In other words the postural control system seems to function appropriately in response to various combinations of sensory deprivation or feedback, but the ability of the system to generate timely and accurately calibrated motor commands may be impaired. Marsden ${ }^{42}$ has argued that the basal ganglia are primarily responsible for the automatic execution of learned motor plans. This hypothesis is supported by the data already quoted, which show various abnormalities in the execution of postural muscle responses. An alternative explanation for the general increase in sway scores is that sensory thresholds for detection of sway are increased in parkinsonian patients. The inability to detect changes in a signal within a limited range is known as 'dead band' in control engineering and has a destabilising effect. Previous studies have suggested that thresholds for detection of body attitude changes are larger in parkinsonian patients, ${ }^{14}{ }^{15}$ which may be relevant in control of upright stance, but there is a lack of other experimental evidence to implicate a threshold detection problem.

In a recent report, a group of slightly more severely affected (HY3, HY4) patients had anterior-posterior sway displacement amplitudes similar to those of young control subjects, both with the platform fixed and with ankles stabilised. ${ }^{11}$ The authors suggest that the low sway amplitudes may have been the result of an increase in the intrinsic mechanical stiffness of the musculoskeletal system, as they found no apparent increase in background EMG, and that this was designed to act as a compensatory mechanism for increased postural instability produced by the disease. These findings directly contradict ours as far as the HY3 subjects are concerned, but the reasons for the discrepancy are not readily apparent.

Contrary to Sheldon's finding of impaired postural control during sway feedback in some elderly subjects, ${ }^{16}$ none of our subjects was adversely affected by this condition. When sway feedback was provided, normal controls and the two groups of patients were able to reduce sway amplitude, both when the platform was fixed and when ankles were stabilised. Although it does not necessarily follow that reduction in body sway implies an improvement in postural performance, particularly during quiet standing on a fixed platform when the balancing task is not challenging, it seems likely that as the task becomes more difficult, reductions in the amplitude of body sway will be associated with improved postural control and a reduced tendency to fall. ${ }^{16}$ The sway feedback provided in our experiments was not, however, simply a short latency visual feedback stimu- 
lus, but rather a visual tracking task that required cognitive processing of visual information to generate estimates of sway amplitude, and conscious generation of appropriate motor commands. The inherent delays present in this process make it unlikely that any improvement in body sway was due to modulation of long loop reflexes, and, in any case, it is not certain that these responses can be elicited by the relatively low stimulus amplitudes occurring in quiet stance.

It is therefore likely that the patients with parkinsonian's disease were able to improve their sway control by using different motor strategies that relied more on conscious effort, and so effectively bypassed their compromised postural reflexes. This is obviously a less automated form of postural control which depends heavily on visual feedback and other external cues to provide an effective motor output. It can also be seen that this is not necessarily an abnormal strategy as our control subjects were able to improve their sway performance in a similar fashion, but this mode of postural control will have limited potential in natural situations where rapidly changing environmental conditions and other subjective distractions will require accurate operation of reflex postural control mechanisms if optimal performance is to be achieved. Mr Hawken was supported by the Wellcome Trust. We would
like to thank Pat Eggington who helped with the patient like to thank Pat Eggington who
assessments and experimental work.

1 Martin JP. The basal ganglia and posture. London: Pitman, 1967:30-5.

2 Chan CWY, Kearney RE, Melville Jones G. Tibialis anterior response to sudden ankle displacements in norma and parkinsonian subjects. Brain Res 1979;173:303-14.

3 Traub MM, Rothwell JC, Marsden CD. Anticipatory postural reflexes in Parkinson's disease and other akineticrigid syndromes and in cerebellar ataxia. Brain rigid syndromes

4 Nutt JG, Nashner LM, Horak FB. Why do Parkinsonian patients fall? Ann Neurol 1983;14:136 (Abstract).

5 Dietz V, Berger W, Horstmann GA. Posture in Parkinson's disease: impairment of reflexes and programming. Ann Neurol 1988;24:660-9.

6 Dick JPR, Rothwell JC, Berardelli A, et al. Associated postural adjustments in Parkinson's disease. $\mathcal{F}$ Neurol Neurosurg Psychiatry 1986;49:1378-85.

7 Knuttson E, Martensson E. Posture and gait in parkinsonian patients. In: Bles W, et al, eds. Disorders of posture and gait. Amsterdam: Elsevier, 1986:217-30.

8 Diener HC, Dichgans J. Long loop reflexes and posture. In: Bles W, et al, eds. Disorders of posture and gait. In: Bles W, et al, eds. Disorders

9 Scholz E, Diener HC, Noth J, Friedemann H, Dichgans J, Bacher M. Medium and long latency EMG responses in leg muscles: Parkinson's disease. $\boldsymbol{f}$ Neurol Neurosurg Psychiatry 1987;50:66-70.

10 Bazalgette D, Zattara M, Bathien N, Bouisset S, Rondot $P$. Postural adjustments associated with rapid voluntary arm movements in patients with Parkinson's disease. Adv Neurol 1986;45:371-4.

11 Horak FB, Nutt JG, Nashner LM. Postural inflexibility in Parkinsonian subjects. $\mathcal{F}$ Neurol Sci 1992;111:46-58

12 Berthoz A, Pozzo T. Intermittent head stabilisation during postural and locomotor tasks in humans. In: Amblard $\mathrm{B}$, et al, eds. Posture and gait: development, adaptation and modulation. Amsterdam: Elsevier, 1988:189-98.

13 Bronstein AM, Hood JD, Gresty MA, Panagi C. Visual control of balance in cerebellar and parkinsonian syndromes. Brain 1990;113:767-79.
14 Danta G, Hilton RC. Judgement of the visual vertical and horizontal in patients with parkinsonism. Neurology 1975;25:43-7.

15 Proctor F, Riklan M, Cooper IS, Teuber HL. Judgement of visual and postural vertical by parkinsonian patients. Neurology 1964;14:287-93.

16 Sheldon JH. The effect of age on the control of sway. Geront Clin 1963;5:129-38.

17 Nashner LM, Black FO, Wall CIII. Adaptation to altered support and visual conditions during stance: patients with vestibular deficits. $\mathcal{F}$ Neurosci 1982;2:536-44.

18 Hoehn MM, Yahr MD. Parkinsonism: onset, progression, and mortality. Neurology 1967;17:427-42.

19 Siegel S, Castellan NJ. Nonparametric statistics for the behavioural sciences. New York: McGraw Hill, 1988 $118 \mathrm{pp}$.

20 Lishman JR, Lee DN. The autonomy of visual kinaesthesis. Perception 1973;2:287-94.

21 Clement $G$, Jacquin T, Berthoz A. Habituation of postural readjustments induced by motion of visual scenes. In: Igarashi $\mathrm{M}$, et al, eds. Vestibular and visual control on posture and locomotor equilibrium. Basel: Karger, 1991: 99-104.

22 Bronstein AM. Suppression of visually evoked postural responses. Exp Brain Res 1986;63:655-8.

23 Woollacott MH, Shumway-Cook A, Nashner LM. Aging and postural control: changes in sensory organization and muscular coordination. Int $f$ Aging Hum Dev 1986 23:97-114.

24 Lee DN, Lishman JR. Visual proprioceptive control of stance. F Hum Movement Stud 1975;1:87-95.

25 Dornan J, Fernie GR, Holliday PJ. Visual input: its importance in the control of postural sway. Arch Phys Med tance in the control of

26 Paulus WM, Straube A, Brandt T. Visual stabilization of posture: physiological stimulus characteristics and clinical aspects. Brain 1984;107:1143-63.

27 Allum JHJ, Keshner EA, Honegger F, Wuethrich R Disturbance of posture in patients with Parkinson's disease. In: Amblard B, et al, eds. Posture and gait: development, adaptation and modulation. Amsterdam: Elsevier, 1988:245-57.

28 Reichert WH, Doolittle J, McDowell FH. Vestibular dysfunction in Parkinson disease. Neurology 1982;32: 1133-8.

29 St-Cyr GJ, Fender DH. Nonlinearities of the human oculomotor system: gain. Vision Res 1969;9:1235-46.

30 Rascol O, Clanet M, Montastruc JL, et al. Abnormal ocular movements in Parkinson's disease: evidence for involvement of dopaminergic mechanisms. Brain 1989 112:1193-214.

31 Takemori S, Maeda T, Seki Y, Aiba T. Vestibular compensation after sudden loss of inner ear or vestibula nerve functions. Acta Otolaryngol (Stockh) Suppl 1984;406:91-4.

32 Black FO, Schupert CL, Peterka RJ, Nashner LM. Effects of unilateral loss of vestibular function on the vestibuloocular reflex and postural control. Ann Otol Rhinol Laryngol 1989;98:884-9.

33 Hytonen M, Pyykko I, Aalto H, Juhola M, Ramsay H. Vestibulo-ocular and vestibulo-spinal reflexes in evaluaVestibulo-ocular and vestibulo-spinal reflexes in evalua-
tion of vestibular lesions. Acta Otolaryngol (Stockh) 1989;468:231-4.

34 Regan D, Maxner C. Orientation-selective visual loss in patients with Parkinson's disease. Brain 1987;110 415-32.

35 Bodis-Wollner I, Marx MS, Mitra S, Bobak P, Mylin L Yahr M. Visual dysfunction in Parkinson's disease: loss of spatiotemporal contrast sensitivity. Brain 1987;110:1675-98.

36 Bulens C, Meerwaldt JD, Van der Wildt GJ. Effect of stimulus orientation on contrast sensitivity in Parkinson's disease. Neurology 1988;38:76-81.

37 van Asten WNJC, Gielen CCAM, Denier van der Gon JJ. Postural adjustments induced by simulated motion of differently structured environments. Exp Brain Res differently struct

38 Stern Y, Mayeux R, Rosen J, Ilson J. Perceptual motor dysfunction in Parkinson's disease: a deficit in sequential and predictive voluntary movement. $f$ Neurol Neurosurg Psychiatry 1983;46:145-51.

39 Cooke JD, Brown JD, Brooks VB. Increased dependence on visual information for movement control in patients with Parkinson's disease. Can $\mathcal{f}$ Neurol Sci 1978;5: 413-5.

40 Flowers $\mathrm{K}$. Lack of prediction in the motor behaviour of parkinsonism. Brain 1978;101:35-52.

41 Crawford TJ, Henderson L, Kennard C. Abnormalities of nonvisually-guided eye movements in Parkinson's disease. Brain 1989;112:1573-86.

42 Marsden CD. The mysterious motor function of the basal ganglia: The Robert Wartenberg Lecture. Neurology ganglia: The Ro 